\title{
Conformational Changes of Immobilized Polythymine due to External Stressors Studied with Temperature-Controlled Electrochemical Microdevices
}

Ramya Vishnubhotla, Christopher B. Montgomery, Kristen L. Steffens, Steve Semancik

Biomolecular Measurement Division, National Institute of Standards and Technology, Gaithersburg, MD 20899, USA

Corresponding author: Ramya Vishnubhotla, rvi@sas.upenn.edu 


\section{Supporting Information}

\section{XPS Data}

X-ray Photoelectron Spectroscopy (XPS) was used to characterize the immobilized polyT as a function of concentration in order to better understand the surface packing density of the species.

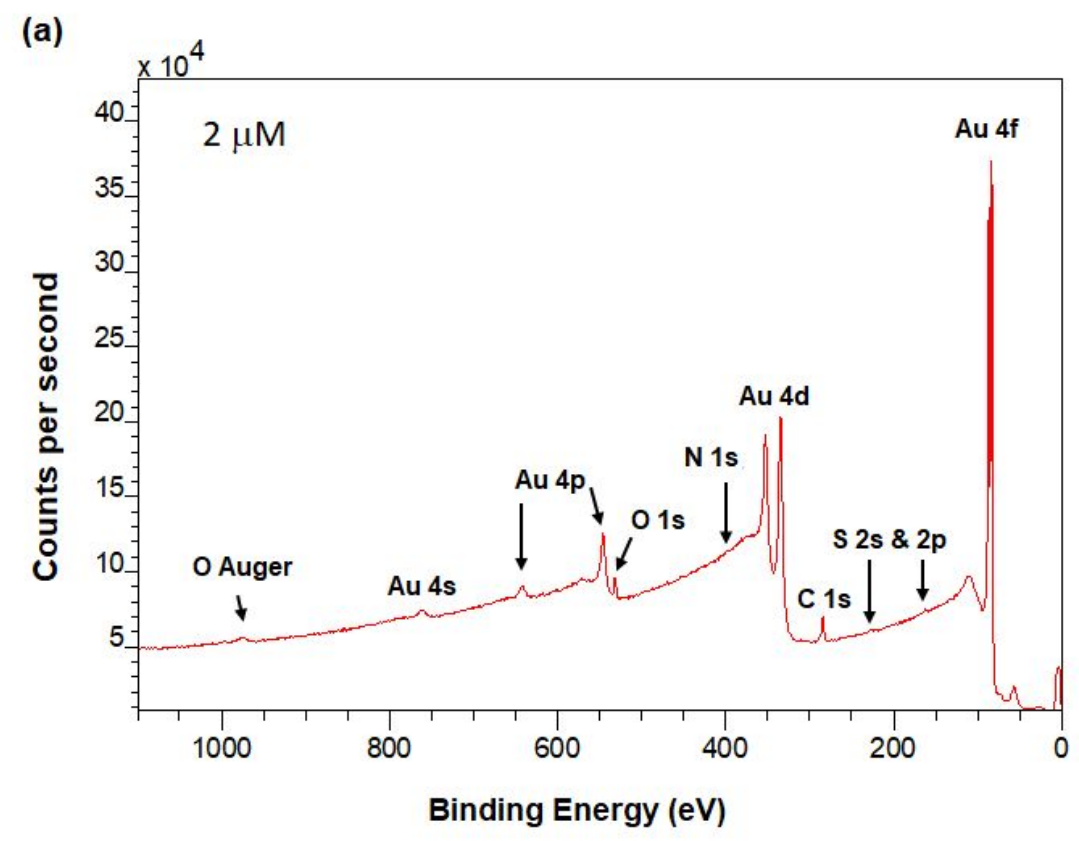


(b)

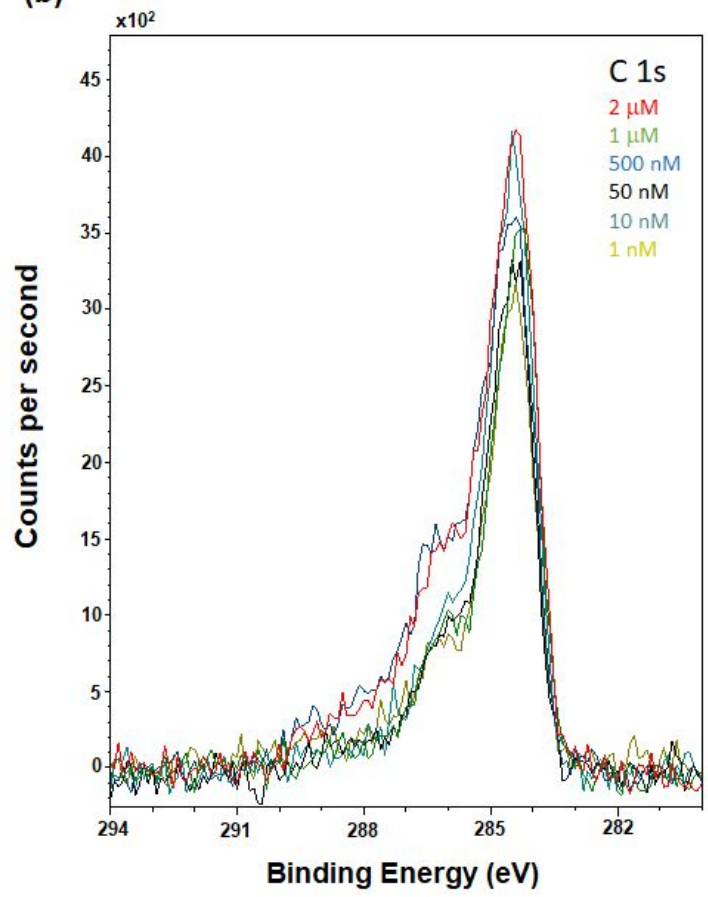

(d)

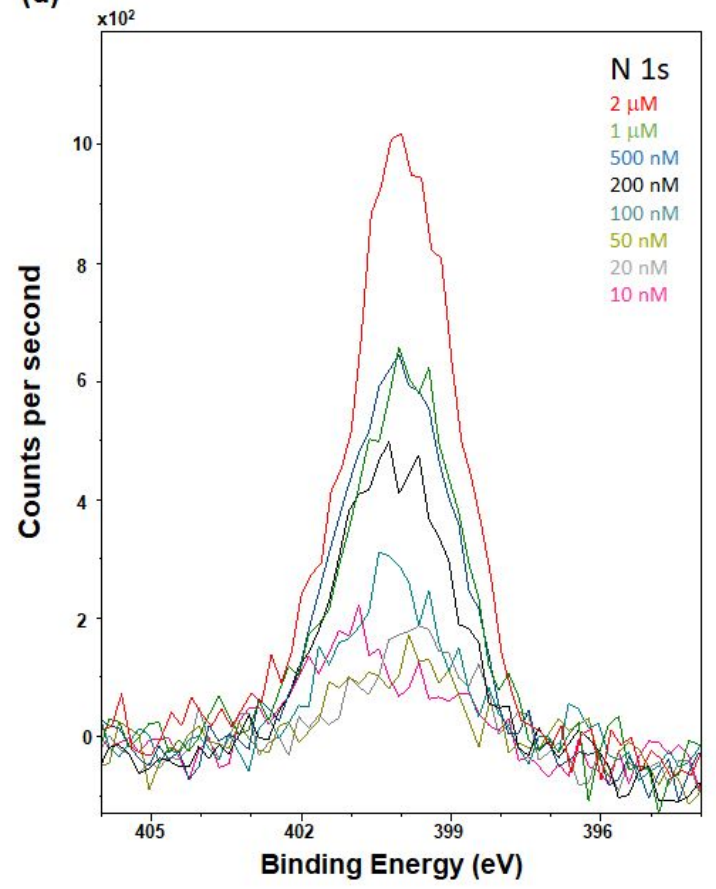

(c)

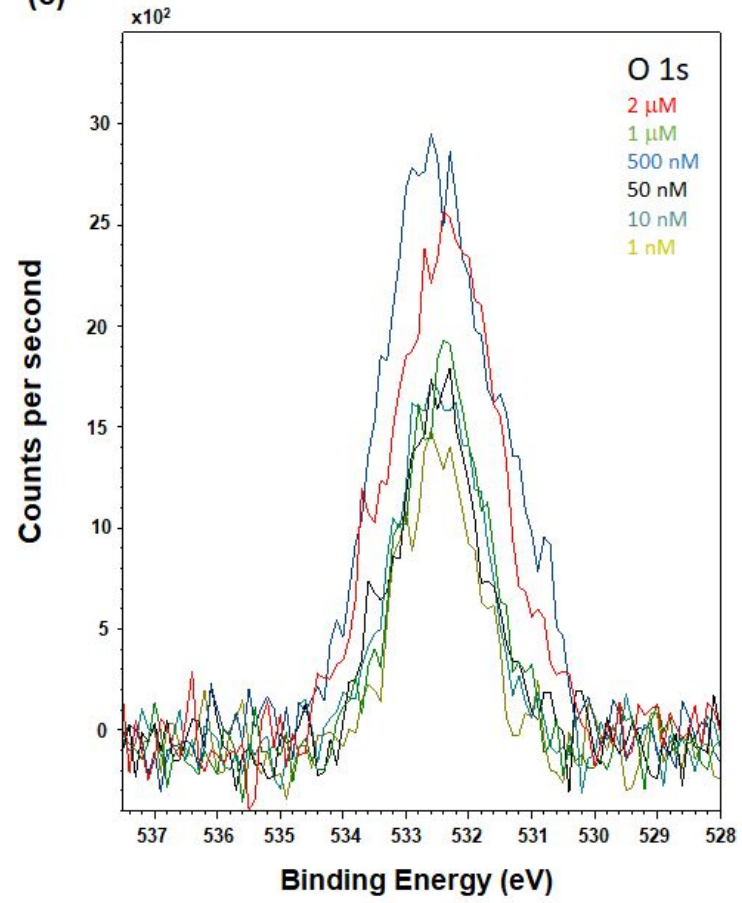

(e)

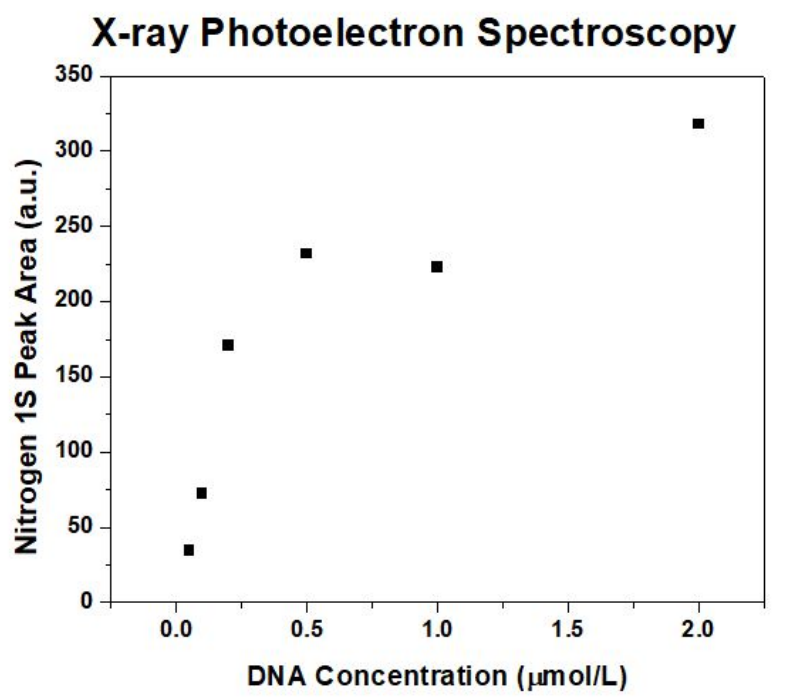

Figure S1. X-ray Photoelectron Spectroscopy (XPS) of polythymine with SAM on Au for: (a) wide scan spectrum, (b) carbon ( $\mathrm{C}$ 1s region), (c) oxygen ( $\mathrm{O}$ 1s region), (d) nitrogen ( $\mathrm{N}$ 1s region), and (e) plot of DNA concentration versus $\mathrm{N}$ 1s signal for 20-mer polythymine over a range of DNA solution concentrations indicates the relative quantity of DNA present on the surface. Samples included a self-assembled monolayer of 6-mercaptohexanol and were formed 
on macro-substrates with of $\sim 200 \mathrm{~nm} \mathrm{Au}$ on $\mathrm{SiO}_{2}$. Spectra were collected using a Kratos* Analytical Axis-Ultra DLD X-ray Photoelectron Spectrometer with a $150 \mathrm{~W}$, monochromated Al $\mathrm{K} \alpha$ source and electrons were detected normal to the sample surface with a $160 \mathrm{eV}$ pass energy, $0.2 \mathrm{eV}$ step size, and $300 \times 700 \mu \mathrm{m}^{2}$ nominal collection area. To improve the signal to noise ratio, each N 1s region scan was averaged for 96 minutes. Subtraction of a linear background was performed using CasaXPS software* (v. 2.3.1PR1.0) prior to calculation of peak areas.

* Any mention of commercial products within NIST web pages is for information only; it does not imply recommendation or endorsement by NIST.

\section{Electrochemical Control Program and Data Collection Methods}

A custom platform was created to control both the temperature program and the electrochemical workstation for a two-device array. Due to each device having slightly different resistances and the existence of slight variabilities between Peltier modules, each device was controlled independently. A proportional integration derivative (PID) controller was incorporated in the software program to control the voltage applied to the thermoelectric module via feedback from the PRT. Standard optimization procedures were performed to determine the proportional, integral, and derivative coefficients for each ramp, placing a priority on roughly minimizing temperature overshoot (at the cost of increased equilibration time). The temperature was controlled by a PID with fixed temperature set points. The PID program involved incubation of

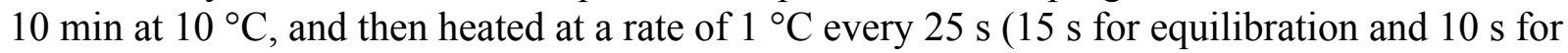
electrochemical analysis).

At each temperature set point, a square wave voltammetric (SWV) scan was performed, voltage window of $-0.2 \mathrm{~V}$ to $-0.7 \mathrm{~V}$ (varied slightly between devices), with a $0.001 \mathrm{~V}$ interval, 60 $\mathrm{Hz}$ frequency and $0.025 \mathrm{~V}$ amplitude. Baseline correction was performed by fitting a straight line to the two lowest points on either side of the monitored MB peak and then subtracting the baseline from the peak current to measure the peak height. All data were plotted in Origin* (OriginPro 2016, OriginLab Corp., Northampton, MA, USA).

Initially, each salt concentration was tested in its own single run (Old Method). While these results were promising, this approach did not preclude device-to-device or run-to-run variations in monitored current when comparing the heating/cooling profiles of the polyT with different $\mathrm{NaCl}$ concentrations. Additionally, the method typically required several days (due to sample preparation time for each run) to test multiple salt solutions and was therefore not timeefficient. Later, a new experimental method (New Method) described in the main text and employed for all data, was used by sequentially measuring the melting profiles for varying salts (seven concentrations), beginning with the lowest salt concentration $(20 \mathrm{mmol} / \mathrm{L} \mathrm{NaCl})$ and continuing to the highest $(1,000 \mathrm{mmol} / \mathrm{L} \mathrm{NaCl})$. Figure $\mathrm{S} 2$ compares results for these two methods. 


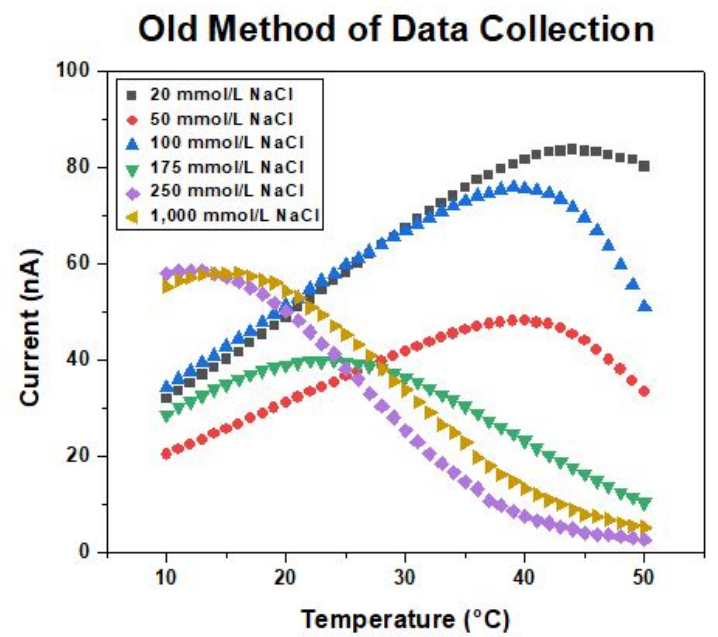

(a)

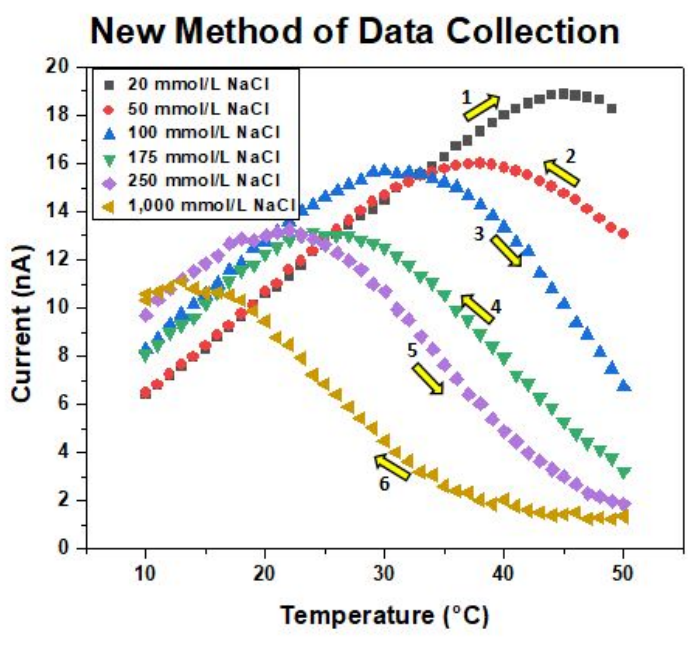

(b)

Figure S2. (a) Initial method of data collection for the 20 mer polyT, where each concentration was done with a single salt concentration on a single day. This method did not preclude deviceto-device variations and was therefore inferior to the newer data collection method; (b) data illustrating the collection method used for this study, again shown here for a 20 mer polyT, where salt concentrations were pipetted out/pipetted in serially between ramp steps. The yellow arrows denote the ramp number and direction (heating/cooling). Note that the data in S2a took a minimum of sixteen hours to acquire, while the data in S2b took approximately three hours to acquire. Peak current differences between these figures and those in the body are most likely due to variations in devices and degradation of devices over a period of time (made more prominent over several months from experimental inactivity due to COVID-19).

\section{Square Wave Voltammetry}



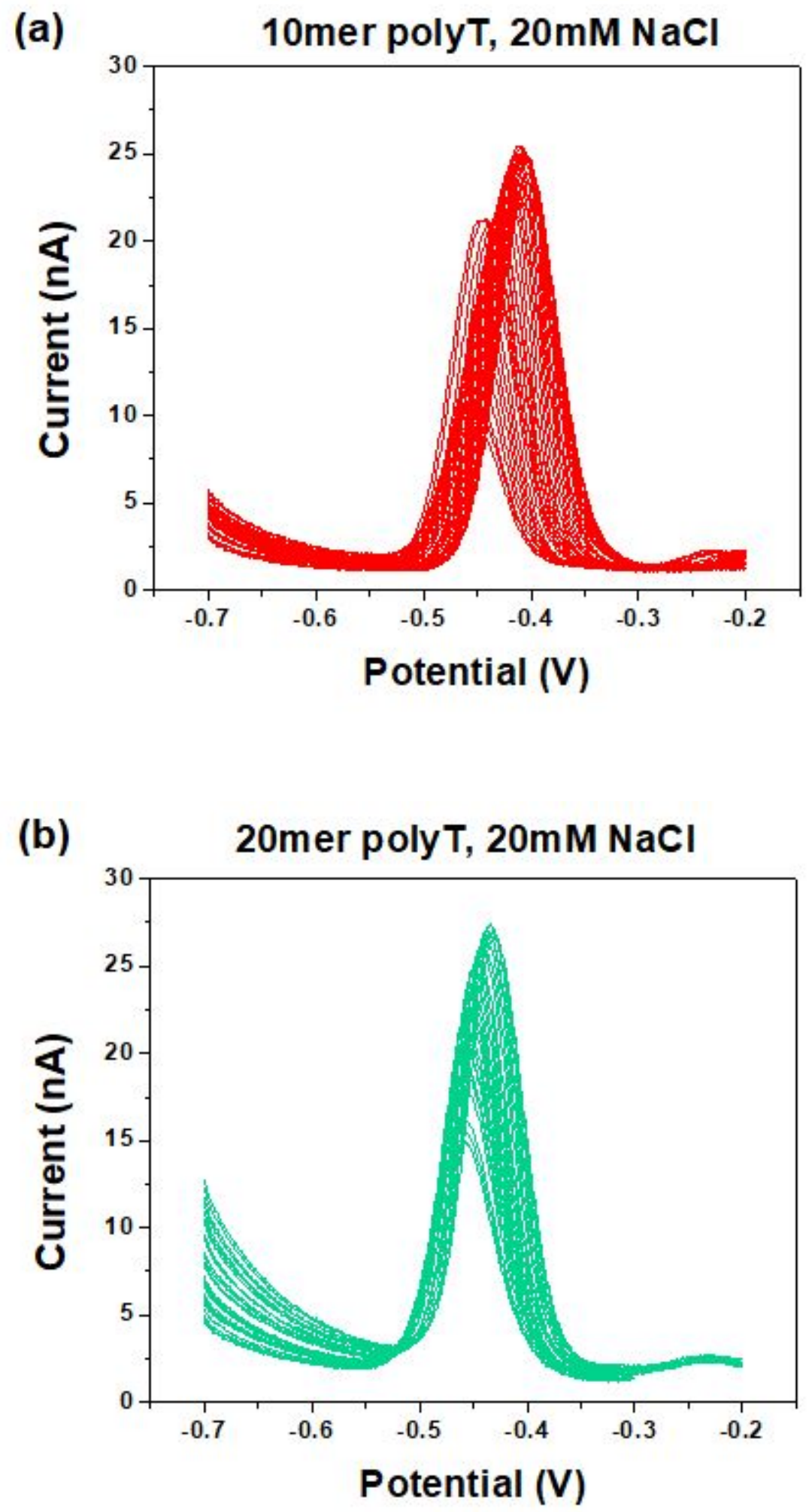


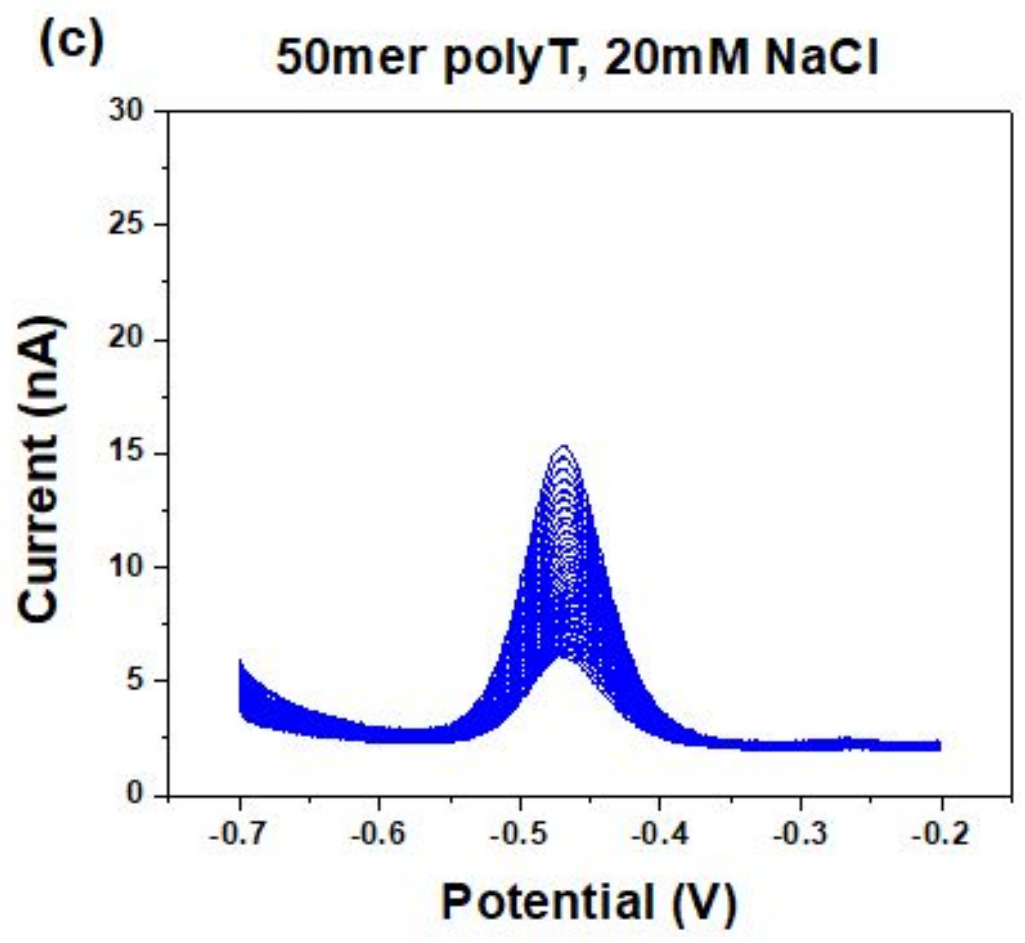

Figure S3. Square-wave voltammetry plots of a (a) 10-mer polyT, (b) 20-mer polyT and (c) 50mer polyT, each at an ionic concentration of $20 \mathrm{mM} \mathrm{NaCl}$. In each instance, the peak current occurs around a voltage of $-0.45 \mathrm{~V}$, with the main difference being the current levels.

\section{Signal Reversibility and Reproducibility}

The following two figures provide results indicating the level of reversibility for the data collection approach, and the reproducibility of acquired data, respectively.

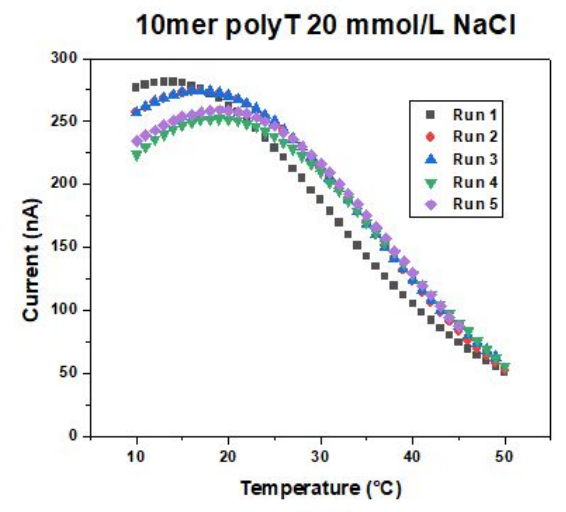

(a)

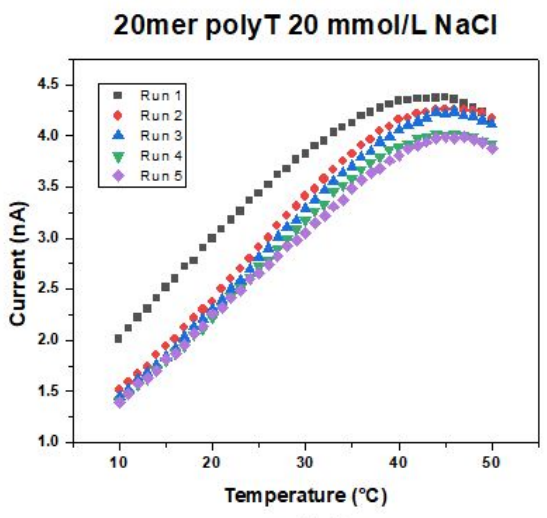

(b)

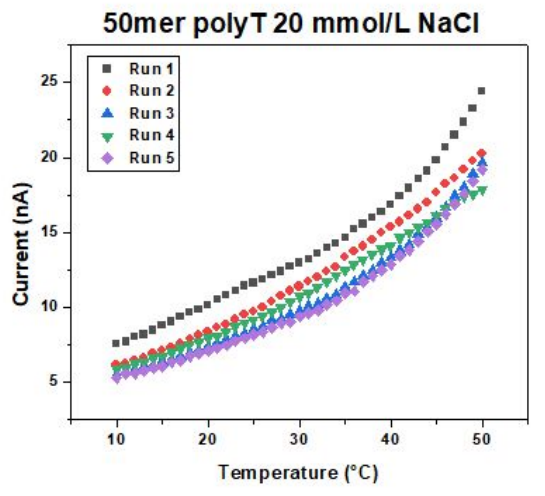

(c) 
Figure S4. Initial studies in a PBS solution of $20 \mathrm{mmol} / \mathrm{L} \mathrm{NaCl}$ for (a) five sequential runs of heating and cooling (heating, cooling, heating, cooling, heating) of a 10mer polyT strand, and (b) five sequential runs of heating and cooling (heating, cooling, heating, cooling, heating) of a 20mer polyT strand, and (c) five sequential runs of heating and cooling (heating, cooling, heating, cooling, heating) of a 50mer polyT strand, with the same PBS solution. Notice the reversibility of the runs, where the old PBS solution was exchanged for fresh solution at the end of each run, regardless of ramp up or down. There is a possibility of slight desorption of the DNA between Runs 1 and 2, although the data is fairly consistent after this point. Peak current value differences between these figures and those in the main text are most likely due to variations in devices and degradation of devices over a period of time (made more prominent over several months from experimental inactivity due to COVID-19). Nevertheless, the consistent trends allow a semi-quantitative study of the polyT system under variable salt and temperature conditions using the more rapid data collection approach.

10 mer polyT Trial 2

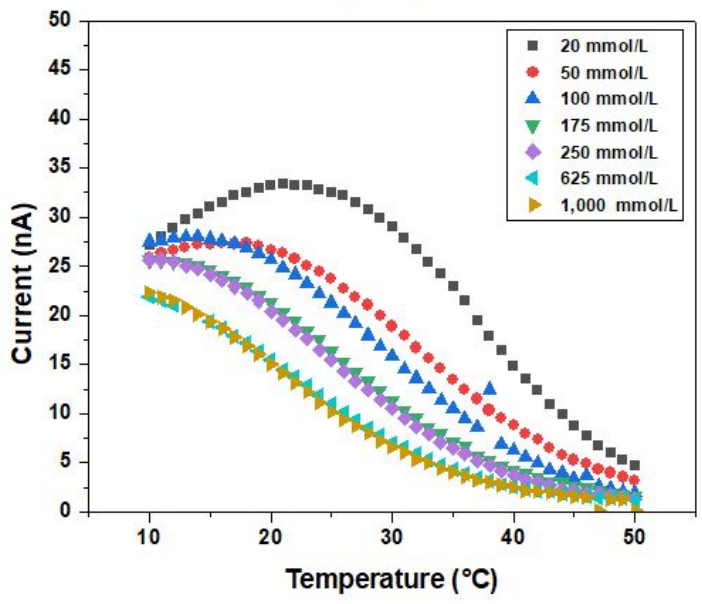

(a)

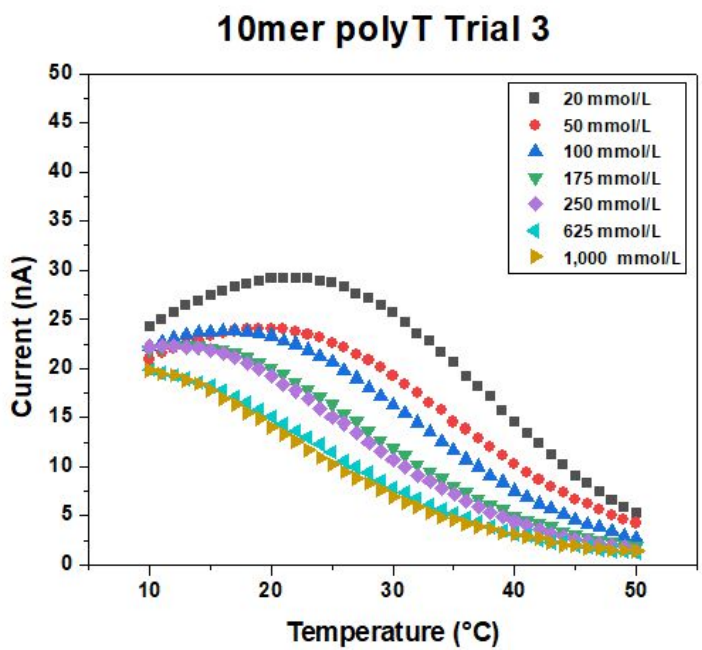

(b) 


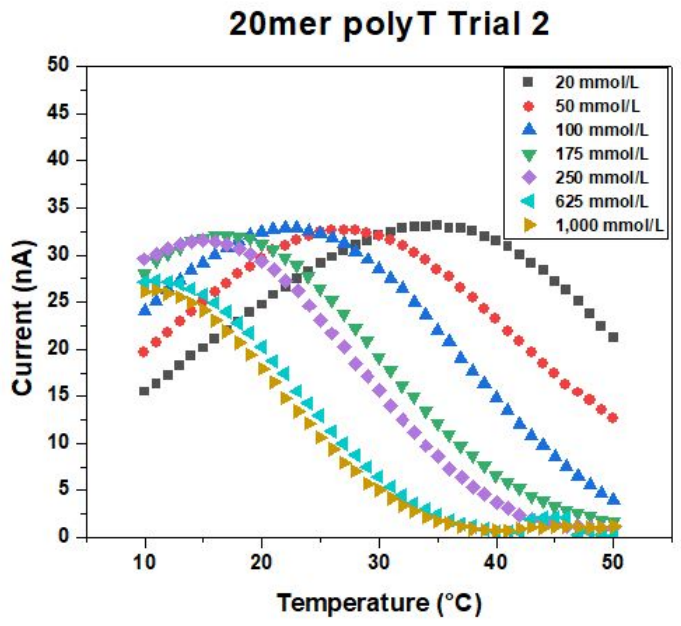

(c)

50mer polyT Trial 2

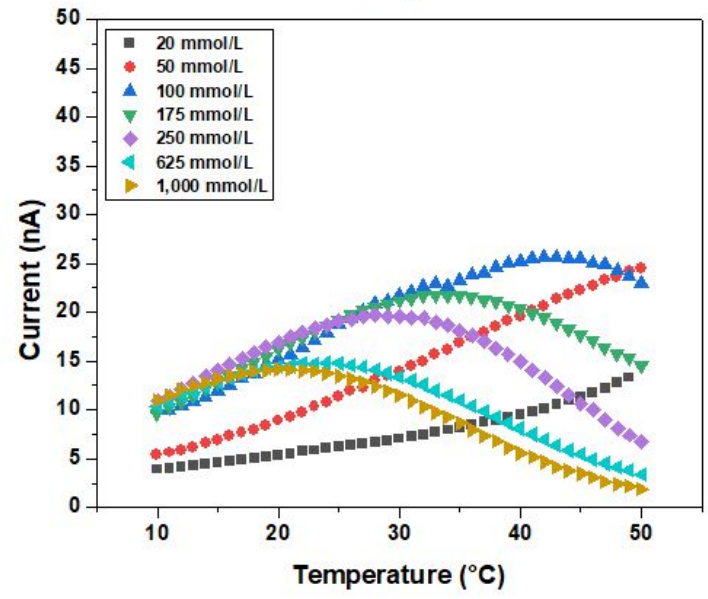

(e)

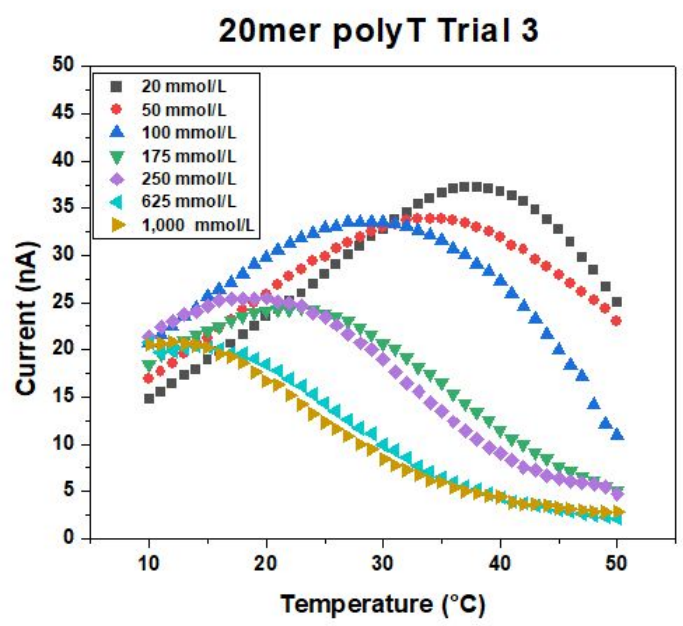

(d)

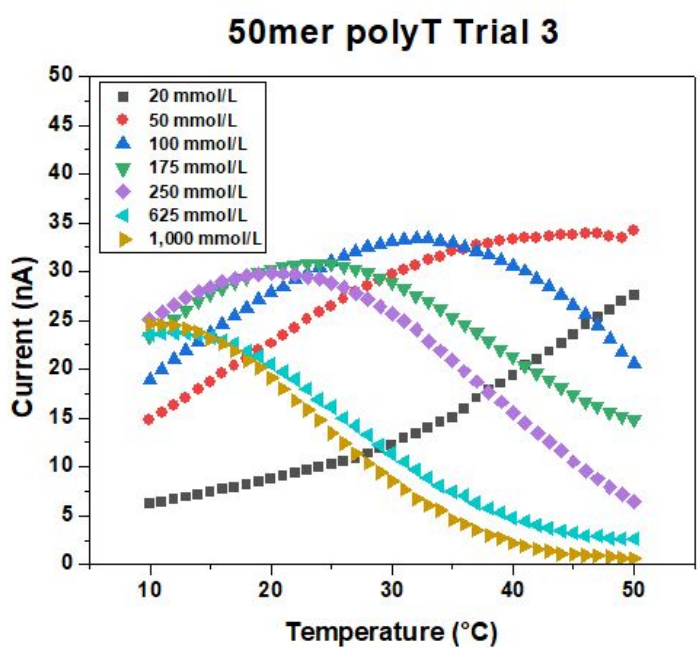

(f)

Figure S5. Additional two trial runs of the 10mer polyT (a and b), the 20mer polyT (c and d) and the 50mer polyT (e and f) strands, that go with Figs. 4a, 5a and 6 a, respectively. We note that although there are slight variations in current, most of the data collected here was done within a short span of time (one month) to avoid, as much as possible, current changes that could arise from degradation of the devices or their surfaces over time. 